\title{
Avaliação do grau de prontidão em gerenciamento de portfólio de projetos: Estudo de caso da Secretaria de Segurança Pública do Estado da Bahia - Brasil
}

\author{
Evaluation of the readiness level in project portfolio management: Case study of the Public \\ Security Department of the State of Bahia - Brazil \\ Evaluación del grado de prontitud en la gestión de portafolio de proyectos: Estudio de caso de la \\ Secretaría de Seguridad Pública del Estado de Bahía - Brasil
}

Recebido: 22/04/2021 | Revisado: 30/04/2021 | Aceito: 21/06/2021 | Publicado: 04/07/2021

\author{
Roberto Otuzi de Oliveira \\ ORCID: https://orcid.org/0000-0002-8321-2863 \\ Centro Universitário Dinâmica das Cataratas, Brasil \\ E-mail: otuziroberto@gmail.com \\ Rodrigo Florencio da Silva \\ ORCID: https://orcid.org/0000-0002-9644-7645 \\ Instituto Politécnico Nacional, México \\ E-mail: rflorencio@ipn.mx
}

\begin{abstract}
Resumo
Este artigo analisa o grau de prontidão em gerenciamento de portfólio de projetos na Secretaria de Segurança Pública do Estado da Bahia, Brasil. O estudo utilizou o modelo PPM Ready (Project portfolio management ready) proposto por Norberto Almeida. Esse modelo analisa o grau de prontidão em gerenciamento de portfólio de projetos por meio de três passos, avaliando quatro pilares. As organizações são estruturadas pelas suas operações contínuas e por seus projetos, que são os meios pelos quais uma ideia é transformada em produto, serviço ou solução tangível. Mas o nível que utilizam e têm conhecimento do gerenciamento de portfólio de projetos variam. Um maior amadurecimento em gerenciamento de portfólio de projetos proporciona custos menores que os previstos, prazos menores com sucesso no encerramento do projeto. Quanto mais madura for uma organização, maior será a taxa de sucesso de seus projetos. Para alcançar a maturidade, existe um esforço contínuo que geralmente leva alguns anos até que os resultados alcançados reflitam uma taxa maior de sucesso nos resultados dos projetos. Vários modelos de avaliação de maturidade em gerenciamento de projetos foram criados para verificar o quanto as organizações estão fazendo para melhorar seu desempenho. A maioria deles avalia o nível de gerenciamento de projetos e há falta dos outros níveis, como o gerenciamento de portfólio. A avaliação da maturidade em organizações que implementaram o gerenciamento de portfólio é um tópico bastante recente e não tem sido discutido na academia em profundidade, principalmente no setor público.
\end{abstract}

Palavras-chave: Maturidade; Gerenciamento de projetos; Gerenciamento de portfólio.

\begin{abstract}
This paper analyzes the degree of readiness in project portfolio management at the Public Security Secretariat of the State of Bahia, Brazil. The study used the PPM Ready model (Project portfolio management ready) proposed by Norberto Almeida. This model analyzes the degree of readiness in project portfolio management through three steps, evaluating four pillars. Organizations are structured by their continuous operations and projects, which are the means by which an idea is transformed into a tangible product, service or solution. But the level they use and are aware of project portfolio management varies. Further maturing in project portfolio management provides lower-than-expected costs, shorter deadlines with successful project closure. The more mature an organization is, the higher the success rate of its projects. To reach maturity, there is a continuous effort that usually takes a few years before the results achieved reflect a higher rate of success in the results of the projects. Several project management maturity assessment models have been created to see how much organizations are doing to improve their performance. Most of them evaluate the level of project management and there is a lack of other levels, such as portfolio management. The assessment of maturity in organizations that have implemented portfolio management is a very recent topic and has not been discussed in depth in academia, especially in the public sector.
\end{abstract}

Keywords: Maturity; Project management; Portfolio Management.

\section{Resumen}

Este artículo analiza el grado de prontitud en la gestión de portafolio de proyectos en la Secretaría de Seguridad Pública del Estado de Bahía, Brasil. El estudio utilizó el modelo PPM Ready (Project portfolio management ready) propuesto por Norberto Almeida. Este modelo analiza el grado de prontitud en la gestión de portafolio de proyectos a través de 
tres pasos, evaluando cuatro pilares. Las organizaciones están estructuradas por sus operaciones continuas y por sus proyectos, que son el medio por el cual una idea se transforma en un producto, servicio o solución tangible. Pero el nivel que utilizan y tienen conocimientos sobre la gestión de portafolio de proyectos varía. Una mayor madurez en la gestión de portafolio de proyectos proporciona costos más bajos de lo esperado, plazos de entrega más cortos para un cierre exitoso del proyecto. Cuanto más madura es una organización, mayor es la tasa de éxito de sus proyectos. Para alcanzar la madurez, hay un esfuerzo continuo que suele tardar unos años hasta que los resultados alcanzados reflejan una mayor tasa de éxito en los resultados del proyecto. Se crearon varios modelos de evaluación de la madurez de la gestión de proyectos para ver cuánto están haciendo las organizaciones para mejorar su desempeño. La mayoría de ellos evalúan el nivel de gestión de proyectos y faltan otros niveles como la gestión de portafolio. La evaluación de la madurez en las organizaciones que han implementado la gestión de portafolio es un tema muy reciente y no se ha discutido en profundidad en la academia, especialmente en el sector público.

Palabras clave: Madurez; Gestión de proyectos; Gestión de Portafolio.

\section{Introdução}

Toda organização quer alcançar a excelência em projetos. Usar o gerenciamento de projetos, mesmo que por um período prolongado de tempo, não é uma condição suficiente para atingir a excelência. A base para alcançar a excelência no gerenciamento de projetos é melhor descrita pelos modelos de maturidade em gerenciamento de projetos, que são compostos por etapas que descrevem a diferença nos níveis de maturidade no gerenciamento de projetos (Kerzner, 2017).

Ao longo dos últimos anos, muitas organizações adotaram uma abordagem orientada para projetos tanto para processos de entrega externa quanto para processos de controle interno. Esta decisão tem impactos positivos na organização, já que sistematiza o trabalho que está sendo entregue (Miklosik, 2015).

A maturidade no gerenciamento de projetos é um fator de sucesso cada vez mais importante para todos os tipos de organização, especialmente organizações que lidam com uma variedade de projetos, programas e portfólios (Bushuyev; Wagner, 2014).

No dicionário Michaelis (2018), a palavra maturidade é definida como o grau de qualidade e desenvolvimento que determinada organização possa atingir, indicando que possa haver um desenvolvimento de um nível de capacidade para um nivel mais elevado. Em outras palavras, a maturidade é a qualidade ou o estado de ser maduro.

A base para alcançar a excelência no gerenciamento de projetos é melhor descrita pelos Modelos de Maturidade na Gestão de Projetos, que são compostos por estágios que descrevem a diferença nos níveis de maturidade no gerenciamento de projetos (De Souza; Gomes, 2015).

Toda organização quer alcançar a excelência em projetos. A base para alcançar a excelência no gerenciamento de projetos é melhor descrita pelos Modelos de Maturidade na Gestão de Projetos, que são compostos por estágios que descrevem a diferença nos níveis de maturidade no gerenciamento de projetos (De Souza; Gomes, 2015).

Os modelos de maturidade estão se mostrando úteis porque permitem que indivíduos e organizações avaliem a maturidade de vários aspectos de seu desempenho em relação a benchmarks e priorizem ações de melhoria. Uma organização madura pode ser vista como aquela que é competente para atender às suas necessidades usando abordagens padronizadas (incluindo a revisão contínua do desempenho), enquanto uma organização imatura não tem a implementação desses processos (OGC, 2010).

Pesquisas indicam que as organizações com níveis mais altos de maturidade devem ter sucesso em termos de eficácia e eficiência; assim, eles têm uma vantagem competitiva no mercado (Backlund; Chronéer; Sundqvist, 2014).

Ao longo dos últimos anos, muitas organizações adotaram uma abordagem orientada para projetos tanto para processos de entrega externa quanto para processos de controle interno. Esta decisão tem impactos positivos na organização, já que sistematiza o trabalho que está sendo entregue (Miklosik, 2015).

O objetivo deste trabalho é avaliar o quanto as organizações estão preparadas para atuar no gerenciamento do portfólio de seus projetos através da análise da prontidão adotada por uma organização à luz do modelo de PPM Ready (Project portfolio 
management ready) proposto por Almeida (2017). A organização escolhida é do setor público representada pela Secretaria de Segurança Pública da Bahia, Brasil.

A Secretaria de Segurança Pública da Bahia foi criada pela Lei no 115, de 16 de agosto de 1895, sendo reorganizada e modificada por diversas leis e decretos ao longo dos anos. O Gerenciamento de Portfólio iniciou-se em 2015, com a seleção, priorização e balanceamento do Portfólio de Projetos para compor o Plano Estratégico do Sistema Estadual de Segurança Pública - PLANESP 2016-2015.

\section{Metodologia}

A pesquisa realizada é de caráter descritivo conforme Prodanov e Freitas (2013), pois busca descrever o grau de prontidão no gerenciamento do portfólio de projetos. Neste estudo foram abordadas as características de uma pesquisa qualitativa.

Quanto ao procedimento técnico, a pesquisa utiliza o estudo de caso. De acordo com Yin (2010), o estudo de caso apresenta o entendimento de aspectos específicos de uma determinada organização, ou seja, os resultados obtidos por meio deste método de procedimento são válidos apenas para a organização em análise, que nesta pesquisa estaria restrita apenas ao setor específico da organização avaliada.

A coleta de dados foi realizada através de entrevista realizada com o gerente de projetos da Secretaria de Segurança Pública da Bahia.

A literatura existente contém contribuições limitadas que abordem a maturidade em gerenciamento de projetos com suporte experimental ou relatem problemas importantes relacionados a mensuração ou melhoria dessa maturidade, bem como os resultados obtidos com sua implantação no setor público brasileiro. Há uma consciência da importância de desenvolver procedimentos de trabalho para garantir maior eficiência no setor público, mas este desenvolvimento muitas vezes não é priorizado em relação às tarefas operacionais. A ausência de priorização é uma indicação do potencial para melhorar a gestão de portfólio de projetos.

Para avaliar o nível de maturidade no gerenciamento de portfólio de projetos de foi utilizado o modelo PPM Ready (Project Portfolio Management Ready) proposto por Almeida (2017).

A ferramenta PPM Ready é baseada na web. A avaliação corresponde a três passos simples.

Passo1: Caracterização da organização e do respondente: serão obtidos os dados básicos do respondente e da organização / área sendo avaliada.

Passo 2: Análise da prontidão - Avaliação do estado atual e do estado desejado para cada uma das perguntas do questionário. No total são quarenta questões, sendo dez para cada pilar.

Passo 3: Diagnóstico e plano de ação - Após a avaliação, com base nas respostas de situação atual e situação desejada de cada uma das 40 questões, é apresentado o estado de prontidão da organização quanto aos quatro pilares, informando a organização avaliada - e, para cada pilar, será informado o estado atual e o estado desejado em percentuais, em uma escala de 0 a 100\%, bem como o diagnóstico final e o plano de ação recomendado, ou seja, o que a organização deve fazer para passar do estado atual para o estado desejado (Almeida \& Olivieri Neto, 2015).

Entenda-se como pilar um elemento de sustentação que dará suporte ao processo de elevação da maturidade das organizações em gerenciamento de projetos.

A avaliação de todos os pilares é baseada no ciclo de melhoria contínua, avaliando-se os seguintes níveis: 
1. A organização tem conhecimento sobre o item, mas ainda não fez qualquer iniciativa neste sentido.

2. A organização já tem iniciativas neste sentido, mas os esforços são isolados, não tendo uma padronização na forma que as diversas áreas fazem.

3. A organização já padronizou e executa o item de forma padronizada entre as áreas.

4. A organização já avalia os resultados dos processos, fazendo um benchmarking entre as áreas e outras organizações.

5. A organização utiliza os resultados do benchmarking para melhorar os processos de forma contínua, já estando a boa prática enraizada na sua cultura.

Para avaliar o quanto as organizações estão prontas nos pilares, foram desenvolvidas dez questões por pilar, em um total de quarenta questões, que foram desenvolvidas como base em um referencial teórico consistente e uma pesquisa de campo abrangente.

Ao final da avaliação, a organização terá a situação atual e a situação desejada em termos percentuais ( 0 a 100\%) em cada pilar, bem como um diagnóstico final e um plano de ação detalhado para alcançar a situação desejada em cada pilar.

Pilar pessoas capacitadas: o objetivo desse pilar é analisar o quanto as organizações possuem pessoas capacitadas tanto na parte técnica quanto comportamental para executar os projetos. Assim foram utilizados como base publicações mundialmente conhecidas, o IPMA Competence Baseline v3.0 - ICB (IPMA, 2015) e o PMBOK Guide (PMI, 2017), além da experiencia do autor Norberto Almeida em consultoría, treinamento e gerenciamento de projetos e portfólios.

Pilar metodología de gerenciamento de projetos, programas e portfólio: segundo o PMI (2017), metodologia é um sistema de práticas, técnicas, procedimentos e regras usado pelas pessoas que trabalham em uma disciplina.

Consoante Almeida e Olivieri Neto (2015, P.19) um conjunto de processos e procedimentos precisa ser criado para elevar o grau de utilização das boas práticas em gerenciamento de projetos, programas e portfólio nas empresas, elevando assim o nível de maturidade em gerenciamento destes domínios. Uma metodologia unificada é um passo imprescindível para alcançar os níveis mais altos de maturidade, permitindo que sejam feitas comparações (benchmarking) e que o processo seja continuamente melhorado, através da análise e melhoria dos indicadores de desempenho. Uma metodología de gerenciamento deve informar a sequência de passos que precisa ser seguida, o conjunto de entradas, técnicas e ferramentas e saídas dos processos a serem utilizado, os modelos de documentos e ferramentas a serem utilizados para a gestão de projetos, programas e portfólios.

O modelo de análise de maturidade em gerenciamento de projetos OPM3 do PMI foi a principal referência para a elaboração das questões desse pilar e na experiência do autor Norberto Almeida.

Pilar tecnologia: é fundamental a ver um sistema de informações de gerenciamento de portfólio (SIGP), que consiste de um conjunto de ferramentas e técnicas para reunir, integrar e disseminar os resultados e as informações do gerenciamento de projetos, importantes para a tomada de decisão, que precisa ser cada vez mais rápida e efetiva em um ambiente de alta competitividade.

Consoante PMI (2017) o sistema de informações de gerenciamento de projetos é um sistema de informações que consiste de ferramentas e técnicas usadas para reunir, integrar e disseminar as saídas dos processos de gerenciamento de projetos.

O sistema de informações de gerenciamento de portfólio (SIGP) corresponde a um conjunto de ferramentas computadorizadas para apoiar a gestão do portfólio de projetos.

Pilar governança de portfólio: as estruturas, funções e processos que norteiam as atividades de gerenciamento do projeto para criar um produto, serviço ou resultado único para atender as metas organizacionais, estratégicas e operacionais (PMI, 2017).

Segundo Thiry e Deguirre (2007), a governança e o gerenciamento harmonizado de um número de projetos e outras ações ajudam a alcançar os benefícios de negócio relatados e criam valor para as partes interessadas. As estruturas de governança alinham os objetivos corporativos à entrega dos produtos. 
Uma estrutura de governança é imprescindível para dar poder, priorizar, definir objetivos, fornecer diretrizes e dar suporte às iniciativas de gerenciamento de projetos, programas e portfólio dentro das empresas. Ela é a base para fazermos corretamente o conjunto mais adequado de projetos e programas. "Mais adequado" dependerá da cultura e da natureza da empresa, mas um exemplo seria implementar os projetos com maior retorno estratégico e com um risco aceitável (Almeida e Olivieri Neto, 2015).

Para avaliar o quanto as organizações estão prontas, foram desenvolvidas questões, onde é possível visualizar todas as questões e opções de respostas. Os Quadros 1 a 4 a seguir demonstram o número da questão e o seu objetivo. Cada questão deve ser avaliada com base na situação atual e situação desejada.

Quadro 1. Questões do pilar pessoas capacitadas.

\begin{tabular}{cl}
\hline $\mathbf{N}^{\mathbf{0}}$ da questão & \multicolumn{1}{c}{ Objetivo } \\
\hline 1.1 & Avaliar a prontidão das organizações nas competências técnicas de gerenciamento de projetos. \\
\hline 1.2 & $\begin{array}{l}\text { Avaliar a prontidão das organizações nas competências comportamentais desejadas para o } \\
\text { gerenciamento de projetos. }\end{array}$ \\
\hline 1.3 & $\begin{array}{l}\text { Avaliar a prontidão das organizações nas competências contextuais desejadas para o } \\
\text { gerenciamento de projetos. }\end{array}$ \\
\hline 1.4 & $\begin{array}{l}\text { Avaliar a prontidão das organizações nas competências técnicas das áreas de negócio dos } \\
\text { projetos. }\end{array}$ \\
\hline 1.5 & $\begin{array}{l}\text { Avaliar a prontidão das organizações em relação às certificações em gerenciamento de } \\
\text { projetos. }\end{array}$ \\
\hline 1.6 & $\begin{array}{l}\text { Avaliar a prontidão das organizações em relação ao estabelecimento de metas e avaliação de } \\
\text { desempenho das equipes de projetos. }\end{array}$ \\
\hline 1.8 & $\begin{array}{l}\text { Avaliar a prontidão das organizações no suporte fornecido às equipes de projetos. } \\
\text { projetos oferecidos às áreas que participem de projetos. }\end{array}$ \\
\hline 1.9 & $\begin{array}{l}\text { Avaliar a prontidão das organizações no conhecimento e na capacitação em gerenciamento de } \\
\text { programas. }\end{array}$ \\
\hline 1.10 & $\begin{array}{l}\text { Avaliar a prontidão das organizações no conhecimento e na capacitação em gerenciamento de } \\
\text { portfólio. }\end{array}$ \\
\hline
\end{tabular}

Fonte: Autores.

Quadro 2. Questões do pilar metodologia.

\begin{tabular}{cl}
\hline $\mathbf{N}^{0}$ da questão & \multicolumn{1}{c}{ Objetivo } \\
\hline 2.1 & $\begin{array}{l}\text { Avaliar a abrangência da metodologia de gerenciamento de projetos em relação às áreas de } \\
\text { conhecimento preconizadas pelo PMI. }\end{array}$ \\
\hline 2.2 & $\begin{array}{l}\text { Avaliar a abrangência da metodologia de gerenciamento de programas em relação às áreas de } \\
\text { conhecimento preconizadas pelo PMI. }\end{array}$ \\
\hline 2.3 & $\begin{array}{l}\text { Avaliar a abrangência da metodologia de gerenciamento de portfólios em relação às áreas de } \\
\text { conhecimento preconizadas pelo PMI. }\end{array}$ \\
\hline 2.4 & $\begin{array}{l}\text { Avaliar se a organização cobre todo o ciclo de melhoria contínua de metodologia (padronizar, } \\
\text { medir, controlar e melhorar). }\end{array}$ \\
\hline 2.5 & $\begin{array}{l}\text { Avaliar se a metodologia cobre todos os grupos de processos (iniciação, planejamento, } \\
\text { execução, monitoramento e controle e encerramento. }\end{array}$ \\
\hline 2.6 & $\begin{array}{l}\text { Avaliar a flexibilidade da metodologia de gerenciamento de projetos em relação às diferentes } \\
\text { complexidades de projetos. }\end{array}$ \\
\hline 2.8 & Avaliar o treinamento dos colaboradores na metodologia de gerenciamento de projetos. \\
\hline 2.9 & Avaliar o nível de utilização da metodologia de gerenciamento de projetos. \\
\hline 2.10 & $\begin{array}{l}\text { Avaliar o o nível de informatização da metodologia de gerenciamento de projetos. } \\
\text { interessadas. }\end{array}$ \\
\hline
\end{tabular}

Fonte: Autores. 
Quadro 3. Questões do pilar tecnologia.

\begin{tabular}{cl}
\hline $\mathbf{N}^{0}$ da questão & \multicolumn{1}{c}{ Objetivo } \\
\hline 3.1 & $\begin{array}{l}\text { Avaliar a existência e a integração de um sistema de informações de gerenciamento do } \\
\text { portfólio. }\end{array}$ \\
\hline 3.2 & Avaliar o nível de utilização da tecnologia de apoio ao gerenciamento de projetos. \\
\hline 3.3 & Avaliar a abrangência da tecnologia no apoio ao processo de governança dos projetos. \\
\hline 3.4 & Avaliar a abrangência da tecnologia no apoio ao processo de gestão corporativa dos recursos. \\
\hline 3.5 & $\begin{array}{l}\text { Avaliar a tecnologia em relação ao suporte à gestão do conhecimento e/ou comunicações do } \\
\text { projeto. }\end{array}$ \\
\hline 3.6 & $\begin{array}{l}\text { Avaliar a tecnologia em relação a sua abrangência no apoio ao controle de demandas e fluxo } \\
\text { de acompanhamento dos projetos }\end{array}$ \\
\hline 3.7 & Avaliar a facilidade e a portabilidade da tecnologia disponível para gerenciar os projetos. \\
\hline 3.8 & Avaliar a eficiência e a manutenibilidade da tecnologia disponível para gerenciar os projetos. \\
\hline 3.10 & $\begin{array}{l}\text { Avaliar o treinamento dos colaboradores nas ferramentas computacionais de suporte ao } \\
\text { gerenciamento de projetos. }\end{array}$ \\
\hline & $\begin{array}{l}\text { Avaliar a aceitação dos sistemas de informação de gerenciamento de portfólio implantados } \\
\text { pelas partes interessadas. }\end{array}$ \\
\hline
\end{tabular}

Fonte: Autores.

Quadro 4. Questões do pilar governança.

\begin{tabular}{cl}
\hline $\mathbf{N}^{0}$ da questão & \multicolumn{1}{c}{ Objetivo } \\
\hline 4.1 & $\begin{array}{l}\text { Avaliar a existência de um planejamento estratégico e o alinhamento dos projetos e } \\
\text { programas às estratégias da organização. }\end{array}$ \\
\hline 4.2 & Avaliar a existência de um comité de governança do portfólio. \\
\hline 4.3 & Avaliar a existência de um Project Management Office ou equivalente. \\
\hline 4.4 & Avaliar a existência de uma área para o gerenciamento do portfólio. \\
\hline 4.5 & $\begin{array}{l}\text { Avaliar a utilização de medida de sucesso e indicadores de desempenho dos projetos } \\
\text { e do portfólio. }\end{array}$ \\
\hline 4.6 & $\begin{array}{l}\text { Avaliar a existência de uma Central de Projetos com indicadores de desempenho e } \\
\text { dashboards. }\end{array}$ \\
\hline 4.8 & $\begin{array}{l}\text { Avaliar a existência de um mapeamento de benefícios (medidas de sucesso e } \\
\text { indicadores de desempenho) esperados pelos projetos ou programas. }\end{array}$ \\
\hline 4.9 & $\begin{array}{l}\text { Avaliar a existência de um processo para categorização, seleção, priorização e } \\
\text { balanceamento do portfólio de projetos e programas. }\end{array}$ \\
\hline $\begin{array}{l}\text { Avaliar a existência de um processo de gestão da mudança organizacional para a } \\
\text { gestão do portfólio de projetos e programas. }\end{array}$ \\
$\begin{array}{l}\text { Avaliar a aceitação do processo de governança do portfólio de projetos e programas } \\
\text { pelas partes interessadas. }\end{array}$ \\
\hline
\end{tabular}

Fonte: Autores.

\section{Resultados e Discussão}

A avaliação do estado atual dessa organização demonstra um grau de prontidão de 52\%, o que é considerado médio, mas no processo de melhoria contínua, a organização busca alcançar 94\%. Após o diagnóstico, é fornecido um plano de ação, visando elevar o grau de prontidão da organização do estado atual para o desejado.

\section{Diagnóstico da análise dos pilares do gerenciamento profissional do portfólio:}

Pessoas capacitadas

Estado atual

$35 \%$

Estado desejado $90 \%$
Metodologia Tecnologia

$58 \%$

$98 \%$
$60 \%$

$90 \%$
Governança

Geral

$52 \%$

$95 \%$ 
O pilar pessoas capacitadas obteve o menor índice e as seguintes ações são recomendadas:

Elaborar ou aprimorar um programa de treinamentos dos profissionais nas competências comportamentais, tais como liderança, negociação e resolução de conflitos, realizar a avaliação de desempenho dos colaboradores nestas competências e fornecer coaching e mentoring aos colaboradores para o seu desenvolvimento nos gaps encontrados.

Elaborar/Aprimorar um programa de treinamentos dos profissionais nas competências técnicas de gerenciamento de projetos, tais como escopo, tempo, custos, riscos, realizar a avaliação de desempenho dos colaboradores nestas competências e fornecer coaching e mentoring aos colaboradores para o seu desenvolvimento nos gaps encontrados.

Elaborar/Aprimorar um programa de treinamentos dos profissionais nas competências contextuais, tais como orientação a projetos, programas e portfólio, segurança, meio ambiente e saúde, aspectos legais, realizar a avaliação de desempenho dos colaboradores nestas competências e fornecer coaching e mentoring aos colaboradores para o seu desenvolvimento nos gaps encontrados.

Elaborar/Aprimorar um programa de treinamentos dos profissionais nas competências técnicas necessárias para atuar na área de negócio dos seus projetos, tais como construção civil, telecomunicações, energia, tecnologia da informação e comunicações e realizar a avaliação de desempenho dos colaboradores nestas competências.

Elaborar um modelo para estimular e patrocinar a certificação dos colaboradores em algumas das principais organizações, tais como PMI, IPMA, AXELOS (PRINCE2), etc.

Desenvolver/Aprimorar um processo de definição de metas e avaliação de desempenho na organização associado a reconhecimentos e recompensas, tais como bônus, participação nos resultados para as áreas de projetos e áreas funcionais que atuem em projetos.

Criar/Aprimorar uma área responsável por dar suporte às equipes nas dúvidas e dificuldades, realizando reuniões periódicas e workshops/treinamentos com as equipes com este propósito.

Criar/Aprimorar uma trilha obrigatória de treinamentos para as áreas em fundamentos de gerenciamento de projetos, bem como em outras áreas de conhecimento em gerenciamento de projetos, tais como, escopo, tempo, custos, etc.

Criar/Manter treinamentos sobre gerenciamento de programas na organização, bem como estimular/patrocinar a certificação dos colaboradores em gerenciamento de programas.

Criar/Manter treinamentos sobre gerenciamento de portfólio na organização, bem como estimular/patrocinar a certificação dos colaboradores em gerenciamento de portfólio.

O pilar governança teve as seguintes ações recomendadas:

Desenvolver/ Aprimorar um planejamento estratégico e um processo para alinhar os projetos e programas às estratégias da organização, garantindo que a grande maioria ou todos os projetos e programas estejam alinhados e que exista a classificação de projetos/programas estratégicos e não estratégicos.

Criar/Aprimorar um comitê de governança do portfólio de projetos, que seja o responsável pela tomada de decisão quanto a seleção e priorização de projetos, utilização dos recursos estratégicos da organização, inclusão ou retirada de projetos ao portfólio, etc., garantindo a reunião periódica deste comitê, com a utilização de Sistemas de Informações de Gerenciamento do Portfólio (SIGP) para suporte à tomada decisão.

Criar/Aprimorar uma área para ser responsável pelo gerenciamento do portfólio de projetos e garantir que utilize um processo de governança previamente estabelecido para atuar em grande parte dos projetos da organização, não somente nos estratégicos.

Desenvolver/Aprimorar um processo de definição, coleta, análise e divulgação de medidas de sucesso e indicadores de desempenho dos projetos e do portfólio, para mais de 3 das dimensões: eficiência do projeto (indicadores de prazo, custo e escopo, etc.); impacto no cliente (satisfação do cliente, atendimento aos requisitos, retenção, etc.); impacto no time (indicadores 
de satisfação, retenção, aumento de produtividade, etc.); sucesso direto (indicadores de retorno sobre o investimento, lucratividade, marketshare, etc.); preparar para o futuro (indicadores de sustentabilidade, contribuição social, responsabilidade ambiental, etc.)), com a definição e a revisão periódica das metas de desempenho.

Desenvolver/Aprimorar uma ferramenta computacional para apresentar uma central única de projetos, com indicadores de desempenho e os dashboards atualizados, com possibilidades de filtros e criação de novas visões e tome ações para criar uma cultura enraizada na organização de avaliação do desempenho dos projetos e tomada de decisão.

Desenvolver/Aprimorar um processo para o gerenciamento dos benefícios esperados pelos projetos e/ou programas durante o seu ciclo de vida e após o seu término, garanta a coleta e a disseminação destas informações na organização e a reutilização de bases históricas e lições aprendidas.

Desenvolver/Aprimorar um processo para a definição de categorias de projetos (ex. projeto estratégico ou não, melhoria da eficiência, produtividade, obrigação legal, etc.) e critérios claros para seleção, priorização e balanceamento dos projetos (ex. alinhamento estratégico, valor presente líquido, retorno sobre o investimento, etc.), garanta sua ampla utilização na organização e um processo de acompanhamento dos resultados e revisão periódica da prioridade dos projetos e programas.

Desenvolver/Aprimorar um processo de gestão da mudança organizacional para a gestão do portfólio de projetos e programas.

Desenvolver/Aprimorar mecanismos para aceitação do processo de governança do portfólio de projetos e programas, tais como workshops, vídeos, boletins, uso de redes sociais e apresentações mostrando os benefícios da sua utilização, garantindo a aceitação de mais de $75 \%$ dos envolvidos.

O pilar metodologia teve as seguintes ações recomendadas:

Desenvolver/Aprimorar uma metodologia para gerenciamento de projetos com processos documentados para mais de 8 das 10 áreas de conhecimento: escopo, tempo, custo, qualidade, recursos humanos, comunicação, riscos, aquisições, partes interessadas e integração.

Desenvolver/Aprimorar uma metodologia de gerenciamento de programas com processos documentados para mais de 7 das 9 áreas de conhecimento: comunicação, financeiro, integração, aquisições, qualidade, recursos, riscos, cronograma e escopo.

Desenvolver uma metodologia de gerenciamento de portfólio com processos documentados para as 5 áreas de conhecimento: gerenciamento da estratégia, gerenciamento da governança, gerenciamento do desempenho, gerenciamento da comunicação, gerenciamento dos riscos.

Desenvolver/Aprimorar os processos de medição, controle e implementar a melhoria contínua da metodologia de gerenciamento de projetos através das oportunidades de melhorias encontradas.

Desenvolver/Aprimorar um programa de treinamentos da metodologia de gerenciamento de projetos, e garantir que mais de $75 \%$ dos envolvidos em projetos sejam treinados.

Elaborar/Aprimorar um programa de incentivo a utilização da metodologia de gerenciamento de projetos, garantindo que pelo menos $75 \%$ dos projetos a utilizem.

Desenvolver/Aprimorar mecanismos para aceitação da metodologia de gerenciamento de projetos da organização com esforços contínuos de conscientização neste sentido, tais como workshops, vídeos, boletins, uso de redes sociais e apresentações mostrando os benefícios da sua utilização, garantindo a aceitação de mais de $75 \%$ dos envolvidos.

O pilar tecnologia teve as seguintes ações recomendadas:

Desenvolver/Aprimorar ferramentas para a gestão dos projetos que atendam as principais áreas de conhecimento do gerenciamento de projetos, tais como: escopo, tempo, custo, qualidade, recursos humanos, comunicação, riscos, aquisições, 
partes interessadas e integração, garantindo que estas ferramentas estejam integradas entre si e com outros sistemas corporativos, tais como ERP, CRM, Financeiro, Recursos Humanos, etc.

Desenvolver/Aprimorar ferramentas de apoio ao processo de governança em mais de 5 itens dos a seguir: planejamento estratégico (definição de visão, missão, objetivos e estratégias), central de projetos, seleção e priorização de projetos, cenários de hipóteses, balanceamento dos recursos organizacionais, relatórios de desempenho dos projetos, dashboards com os indicadores de desempenho dos projetos.

Desenvolver/Aprimorar ferramentas de apoio ao processo de gestão corporativa de recursos em pelo menos 4 dos itens a seguir: base de recursos humanos, materiais, equipamentos, infraestrutura, financeiro; disponibilidade e alocação dos recursos; histograma de recursos, visão de uso dos recursos em todos os projetos, timesheet dos recursos nos projetos, integração com a ferramenta de agenda/e-mail da empresa, análise do desempenho dos recursos, busca de recursos por perfil e/ou competências.

Desenvolver/Aprimorar ferramentas computacionais para dar suporte totalmente ao controle de demandas e fluxo de acompanhamento dos projetos, garantindo que os tipos de demandas e fluxo de acompanhamento possam ser alterados sem a ajuda de consultoria e/ou empresas especializadas e que haja um processo implantado de revisão e melhoria contínua destes itens.

Desenvolver/Aprimorar as ferramentas computacionais, para que tenham mais facilidade de uso, possam ser utilizadas desconectadas da rede da organização e tenham portabilidade para smartphones, tablets, etc.

Desenvolver/Aprimorar as ferramentas computacionais para que apresentem desempenho apropriado (ausência de lentidão, travamentos contínuos ou resultados incorretos).

Desenvolver/Aprimorar mecanismos para aceitação das ferramentas computacionais de gerenciamento de projetos da organização, tais como workshops, vídeos, boletins, uso de redes sociais e apresentações, mostrando os benefícios da sua utilização, garantindo a aceitação de mais de $75 \%$ dos envolvidos.

\section{Considerações Finais}

À medida que as organizações continuam crescendo e se desenvolvendo e à medida que o conhecimento do gerenciamento de portfólio de projetos continuam se desenvolvendo, cada vez mais as organizações querem saber como estão em sua própria curva de aprendizado e o que devem fazer para melhorar seu desempenho no gerenciamento de portfólio de projetos. A maturidade do gerenciamento de portfólio avalia a probabilidade da organização de atingir com sucesso os objetivos estratégicos e lhes proporciona vantagem competitiva no mercado. Apesar do papel significativo do gerenciamento de portfólio, não há um modelo integrado avaliando diferentes dimensões de maturidade do portfólio, e poucos modelos presentes têm muitas desvantagens. Esta pesquisa estabeleceu uma a avaliação da prontidão da Secretaria de Segurança Pública da Bahia para gerenciar seu portfólio de projetos, baseado nos quatro pilares do gerenciamento profissional de projetos, propostos por Almeida (2017).

\section{Referências}

Almeida, N. O. (2017). Gerenciamento de portfólio e PMO. FGV Editora.

Almeida, N. O., Olivieri Neto, R. (2015). Gestão profissional do portfólio de projetos: maturidade e indicadores. Brasport.

Backlund, F., Chronéer, D., \& Sundqvist, E. (2014). Project Management Maturity Models - A Critical Review: A Case Study within Swedish Engineering and Construction Organizations. Procedia - Social and Behavioral Sciences, 119, 837-846. doi.org/http://dx.doi.org/10.1016/j.sbspro.2014.03.094

Bushuyev, S. D., Wagner, R. F. (2014). IPMA Delta and IPMA Organisational Competence Baseline (OCB). International Journal of Managing Projects in Business. doi.org/10.1108/IJMPB-10-2013-0049

De Souza, T. F., \& Gomes, C. F. S. (2015). Assessment of maturity in project management: A bibliometric study of main models. In Procedia Computer Science. 55, 92-101. doi.org/10.1016/j.procs.2015.07.012 
Research, Society and Development, v. 10, n. 8, e2010815329, 2021

(CC BY 4.0) | ISSN 2525-3409 | DOI: http://dx.doi.org/10.33448/rsd-v10i8.15329

Dooley, K., Subra, A., \& Anderson, J. (2001). Maturity and its impact on new product development project performance. Research in Engineering Design Theory, Applications, and Concurrent Engineering. 13(1), 23-29. doi.org/10.1007/s001630100003

Holmes S. J., Walsh R. T. (2005). Conducting Effective Project Management Maturity Assessment Interviews. Integrated Management Systems (IMSI TECH), Inc., Ann Arbor, MI. Recuperado de http://www.imsi-pm.com/home/library/conducting_assessment.pdf

International Project Management Association (2015). IPMA Competence Baseline. (4a ed.), IPMA. Recuperado de http://products.ipma.world/wpcontent/uploads/2016/03/IPMA_ICB_4_0_WEB.pdf

Jugdev, K., Mathur, G. (2012). Classifying project management resources by complexity and leverage. International Journal of Managing Projects in Business. 5(1), 105-124. doi.org/10.1108/17538371211192928

Judgev, K., Thomas, J. (2002). Project management maturity models: The silver bullets of competitive advantage. Project Management Journal. 33(4), 4-14

Kerzner, H. (2001). Strategic Planning for Project Management Using a Project Management Maturity Model. Editora John Wiley \& Sons, 2001. Recuperado de http://metalab.uniten.edu.my/ abdrahim/mitm743/strategicforPM.pdf

Kerzner, H. (2017). Gestão de Projetos: As Melhores Práticas. (3a ed.), Bookman.

Miklosik, A. (2015). Improving Project Management Performance through Capability Maturity Measurement. Procedia Economics and Finance. $30,522-530$. doi.org/10.1016/s2212-5671(15)01264-2

Office of Government Commerce (2010). Portfolio, Programme and Project Maturity Model (P3M3): Introduction and Guide to P3M3, The Stationery Office: London. http://miroslawdabrowski.com/downloads/P3M3/OGC\%20branded/P3M3_v2.1_Introduction_and_Guide.pdf

Pajares, J., López, A. (2014). New Methodological Approaches to Project Portfolio Management: The Role of Interactions within Projects and Portfolios. Procedia - Social and Behavioral Sciences. https://doi.org/10.1016/j.sbspro.2014.03.072

Pilkaite, A., Chmieliauskas, A. (2015). Changes in public sector management: Establishment of project management offices - A comparative case study of Lithuania and Denmark. Public Policy and Administration. https://doi.org/10.13165/VPA-15-14-2-10

Prodanov, C. C.; Freitas, E. C. de. (2013). Metodologia do trabalho científico: métodos e técnicas da pesquisa e do trabalho acadêmico. (2a ed.), Feevale.

Project Management Institute (2013). OPM3 Knowledge Foundation. (3a ed.), wn Square.

Project Management Institute (2017). The Guide to the Project Management Body of Knowledge. (6a ed.), Square.

Project Management Institute. (2017a). PMI's pulse of the profession: capturing the value of Project management. Newtown Square.

Project Management Institute (2017b). The Standard for Portfolio Management. (4a ed.), Newtown Square.

Rouse, M. (2007). Capability Maturity Model (CMM) definition, http://searchsoftwarequality.techtarget.com/definition/Capability-Maturity-Model.

Thiry, M., Deguire, M. (2007). Recent developments in project-based organisations. International Journal of Project Management. 25, 649-658 doi.org/10.1016/j.ijproman.2007.02.001

Yin, R, K. (2010). Estudo de caso: Planejamento e métodos. (4a ed.), Bookman. 